\title{
Radiation losses of type-I ELMs during impurity seeding experiments in the full tungsten ASDEX Upgrade
}

\author{
J.C. Fuchs*, M Bernert, T Eich, A Herrmann, P de Marné, B Reiter, \\ and the ASDEX Upgrade Team \\ Max-Planck-Institut für Plasmaphysik, EURATOM Association, Garching, Germany
}

\begin{abstract}
Edge localised modes (ELMs) are a serious concern for plasma facing components in next generation tokamaks. In order to keep the power load on divertor targets at a reasonable level, operation at high heating powers in ASDEX Upgrade with boronised full tungsten walls was possible only with radiative cooling by nitrogen seeding. The key player to reduce the power load is the control of the divertor radiation. This paper investigates radiation losses between and during type-I ELMs in unseeded and nitrogen seeded discharges. The ELM averaged radiation level raises from $60 \%$ of the input power in unseeded discharges to $80 \%$ in nitrogen seeded discharges. The increase of radiation during an ELM is nearly the same for seeded an unseeded discharges, but due to the smaller ELM size in seeded discharges about $40 \%$ of the ELM energy is radiated compared to $20 \%$ in unseeded discharges.
\end{abstract}

JNM keywords: P0600, I0100

PSI-19 keywords: ASDEX Upgrade, ELM, Impurity seeding, Radiation, Power balance PACS: $52.55 . \mathrm{Fa}, 52.55 . \mathrm{Rk}, 52.25 . \mathrm{Vy}$

Corresponding author address:

Boltzmannstr. 2, 85748 Garching, Germany phone: +49 8932991730

fax: +49 8932992580

Corresponding author e-mail: christoph.fuchs@ipp.mpg.de

Presenting author: J. Christoph Fuchs

Presenting autor e-mail: christoph.fuchs@ipp.mpg.de 


\section{Introduction}

Since 2007 all plasma facing components (PFCs) in ASDEX Upgrade are coated with tungsten in order to investigate the plasma wall interaction and its implications in an all tungsten divertor tokamak, i.e. in absence of carbon. With the first boronisation after more than one year of operation with full-W coating, the intrinsic radiation level dropped and the power load to the divertor targets increased such that the power flux could exceed the power limits of a few target tiles with thick vacuum plasma sprayed (VPS) coating. Therefore operation at high heating powers was possible only with radiative cooling, which was achieved by feedbackcontrolled injection of nitrogen using thermoelectric current measurements in the outer divertor. This resulted not only in the desired divertor power load reduction, but also in improved energy confinement [1].

The key player to reduce the power load on the divertor plates is the control of the divertor radiation. The radiation in ASDEX Upgrade discharges is routinely measured by up to 104 gold foil bolometers mounted in several cameras at different poloidal positions in one toroidal position, as well as 192 AXUV photodiodes at two toroidal positions. From the measured line integrals the total radiated power can be determined and the distribution of the radiation emissivity is reconstructed in the divertor and X-point region as well as in the main plasma by deconvolution methods.

\section{Results and discussion}

\subsection{Radiation distribution during nitrogen seeding}

Figure 1 shows a comparison of the distribution of the ELM averaged radiation emissivity in a poloidal cross section of ASDEX Upgrade with unboronised, full tungsten walls for two discharges with 1MA plasma current, 10.75 MW additional heating power (10 MW neutral 
beam injection, $0.75 \mathrm{MW}$ ECRH) and a fractional Greenwald density of $\mathrm{n}_{\mathrm{e}} / \mathrm{n}_{\mathrm{GW}} \sim 0.65$. The first plot is for a reference discharge without any impurity seeding, the second one for a discharge with feedback controlled nitrogen seeding using thermoelectric current measurements in the outer divertor. Also the difference between these two radiation profiles is plotted.

Whereas in the unseeded discharge we find a total radiated power of about $60 \%$ of the input power, the radiation level in the nitrogen seeded discharge increased as expected to a level of about $80 \%$ of the input power, however the distribution of the radiation has changed signifi-

cantly: the increase of the total radiated power is mainly caused by the increased radiation from the X-point and divertor region, which raised from ELM averaged local emissivities of 4-5 $\mathrm{MW} / \mathrm{m}^{3}$ in unseeded discharges to more than $7 \mathrm{MW} / \mathrm{m}^{3}$ in nitrogen seeded discharges, which is comparable to former campaigns with mixed carbon and tungsten PFCs. Correspondingly the integrated radiated power from the $\mathrm{X}$ point and divertor region has increased by more than a factor of two from $5-10 \%$ of the input power to $15-20 \%$. In accordance with the higher radiation the power load on target plates has decreased by up to a factor of 2 .

\subsection{Radiation during type-I ELMs with nitrogen seeding}

Despite the relatively slow time resolution of the foil bolometers of approximately $1 \mathrm{~ms}$ one can investigate radiation energy losses due to type-I ELMs by using the accumulated energy on a bolometer foil instead of the momentarily absorbed power [2]. Figure 2 shows for both the unseeded and the nitrogen seeded discharges from figure 1 a reconstruction of the radiated energy due to a type-I ELM during a $2 \mathrm{~ms}$ interval around the ELM, where the background radiation between ELMs has been subtracted. Integrating the radiation emissivity over the whole plasma volume or parts of it gives the total radiated power and the radiated power e.g. from the divertor or the core plasma. Time traces for both the radiated power between ELMs and during ELMs are shown in figure 3. 
For both the unseeded discharge and the nitrogen seeded discharge the additional radiated energy during a $2 \mathrm{~ms}$ interval around the type-I ELM from figure 2 is about $7 \mathrm{~kJ}$, the higher radiation during ELMs in the nitrogen seeded discharge is mainly caused by the higher background radiation between ELMs. However the distribution of the additional radiated energy is different: In the unseeded discharge the highest emissivities are observed near the inner and outer strike points, whereas in the nitrogen seeded discharges the radiation in the inner divertor is more located at the $\mathrm{X}$ point and towards the main plasma.

Since in the nitrogen seeded discharges the energy loss per ELM is smaller than in unseeded discharges [3], the normalised ELM radiation $\mathrm{E}_{\mathrm{rad}} / \mathrm{E}_{\mathrm{ELM}}$ (with the background radiation subtracted) raises from $20-25 \%$ of the ELM energy in unseeded discharges to values of $30-40 \%$ in nitrogen seeded discharges (figure 4). These values are comparable to those found in former campaigns with mixed carbon and tungsten PFCs [2], and are in line with JET [4,5]. After boronisation of the PFCs the overall radiation level decreased [6], both between and during ELMs, and about 25\% of the ELM energy is found as radiation in nitrogen seeded discharges.

The newly installed AXUV photodiodes allow to measure the plasma radiation with a time resolution of $200 \mathrm{kHz}$ [7]. Therefore, after calibrating their measured line integrals with the absolute values measured by the foil bolometers, even a reconstruction of the development of the radiation distribution during ELMs is possible. Figure 5 shows the time evolution of the divertor radiation during one ELM in a nitrogen seeded discharge with boronised PFCs. In the outer divertor a rapid increase of the radiation near the strike point at the beginning of the ELM is observed, whereas in the inner divertor the radiation from several successive filaments is found well above the strike point. A more detailed analysis of the radiation development during an ELM is in progress [8]. 


\subsection{Energy balance}

Figure 6 shows the energy balance between and during ELMs for unseeded and nitrogen seeded discharges: Plotted are the loss energy during 2ms intervals (radiated energy measured by bolometers, energy load on the divertor targets measured by IR cameras, corrected by those parts of the divertor radiation which are recorded by both bolometers and IR cameras) against the input energy during these $2 \mathrm{~ms}$ intervals (heating energy and change of the MHD energy of the plasma). Although input and loss energies are approximately balanced, both between and during ELMs the loss energy does not sum up to the full input energy, especially during larger ELMs. The main part of the missing energy is probably caused by thermal load on PFCs outside the divertor, which has not been taken into account in this calculation. It was shown that typically about $8 \%$ of the input energy and $25 \%$ of the ELM energy is deposited on non-divertor components [9]. In accordance with the higher radiation less heat flux to targets both in and outside the divertor has been found for the nitrogen seeded discharges.

\section{Summary and Conclusions}

Radiation losses between and during type-I ELMs in ASDEX Upgrade with full tungsten walls have been investigated for unseeded and nitrogen seeded discharges. It has been found that the ELM averaged radiation level is about $60 \%$ of the input power in unseeded discharges and raises up to $80 \%$ in nitrogen seeded discharges, whereas the power load on targets in and outside the divertor is reduced as desired. The increase of the radiation is mainly caused by increased radiation between ELMs, the additional radiation during ELMs is nearly the same for both seeded and unseeded discharges. About 20-25\% of the ELM energy is found as radiation in unseeded discharges and $30-40 \%$ in nitrogen seeded discharges, due to the smaller ELM size. This value is comparable to the values found in former campaigns with mixed carbon and tungsten PFCs. High temporal resolution measurements of the radiation with AXUV di- 
odes show radiation of several successive filaments especially in the inner divertor. Comparing the radiated energy and energy load to target plates with the input power and change of the MHD energy, and taking into account the estimations of the energy being deposited onto main chamber surfaces which was not directly measured [9], one arrives at a reasonably good power balance between and during ELMs.

\section{References}

[1] A Kallenbach et al, Plasma Phys. Control. Fusion 52 (2010), 055002

[2] J.C. Fuchs et al, Journal of Nuclear Materials, 337-339 (2005), 756

[3] O. Gruber et al, Nucl. Fusion 49 (2009), 115014

[4] A. Huber et al, Journal of Nuclear Materials 390-391 (2009) 830-834

[5] J. Rapp et al, Nuclear Fusion 49 (2009), 095012

[6] J.C. Fuchs et al, Europhysics Conference Abstracts 33E (2009), P1-147

[7] B. Reiter, PhD Thesis, LMU München (2010)

[8] M. Bernert, Diploma Thesis, TU München (2010)

[9] A. Herrmann et al, Plasma Phys. Control. Fusion 46 (2004), 971-980 


\section{Figure captions}

\section{Figure 1:}

Distribution of the ELM averaged radiation emissivity in a poloidal cross section of ASDEX Upgrade for two discharges with similar plasma parameters: one reference discharge without impurity injection, and one discharge with nitrogen seeding. The picture on the right hand side shows the difference between these two distributions, where positive values denote more radiation in the seeded discharge.

Figure 2:

Distribution of the additional radiated energy due to a type-I ELM during a 2ms interval around the ELM for the unseeded and the nitrogen seeded discharges from figure 1, averaged over several ELMs.

\section{Figure 3:}

Time traces of the radiated power between (top) and during (bottom) ELMs for an unseeded (left) and nitrogen seeded (right) discharge. Plotted are the input power (black, heating power $\left.-\mathrm{dW}_{\mathrm{MHD}} / \mathrm{dt}\right)$, the total radiated power, and the radiated power in the core plasma and in the divertor.

\section{Figure 4:}

Dependence of the normalised ELM radiation $\mathrm{E}_{\mathrm{rad}} / \mathrm{E}_{\mathrm{ELM}}$ (where $\mathrm{E}_{\mathrm{rad}}$ is the radiated energy due to a type-I ELM) of the ELM energy $\mathrm{E}_{\mathrm{ELM}}$ for unseeded discharges, nitrogen seeded discharges and nitrogen seeded discharges with boronised plasma facing components. Plotted are mean values for different shots with error bars.

\section{Figure 5:}

Development of the radiation during a type-I ELM. In the upper part contour plots of the line integrals of the AXUV diodes in the inner and outer divertor are shown, the position of these line of sights is shown in the inlay in the lower graph. The dotted line indicates the position of 
the strike points. The lower graph shows the total radiated power and the radiated power from the divertor due to the ELM (where the background radiation has been subtracted).

\section{Figure 6:}

Energy balance between ELMs (top) and during ELMs (bottom) for unseeded (left) and nitrogen seeded (right) discharges. Plotted is the loss energy (radiated energy and energy load onto divertor target plates) during $2 \mathrm{~ms}$ intervals against the input energy (heating energy during the $2 \mathrm{~ms}$ interval and change of $\left.\mathrm{W}_{\mathrm{MHD}}\right)$. 
w/o $\mathrm{N}_{2}$

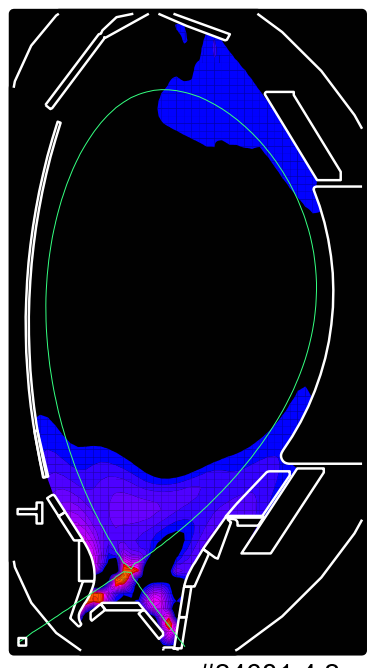

\#24681 4.2s with $\mathrm{N}_{2}$

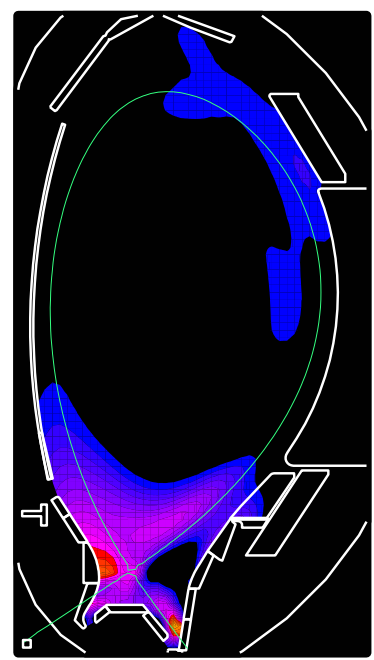

\#24682 4.2s
8.0

$6.4 \sum_{\sum}^{\text {लह }}$

4.8

3.2

1.6

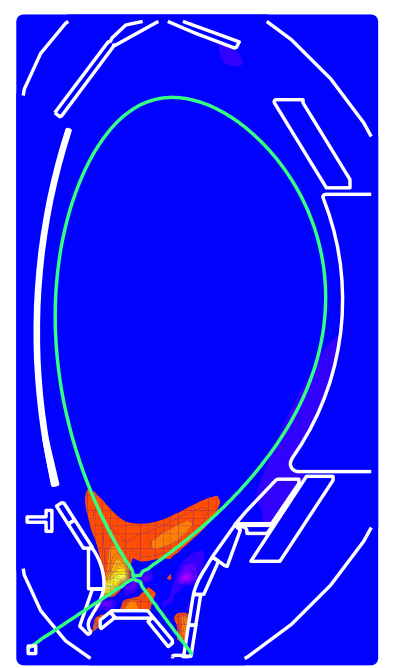

5

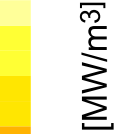

2.5

$-2.5$

Figure 1: Distribution of the ELM averaged radiation emissivity in a poloidal cross section of ASDEX Upgrade for two discharges with similar plasma parameters: one reference discharge without impurity injection, and one discharge with nitrogen seeding. The picture on the right hand side shows the difference between these two distributions, where positive values denote more radiation in the seeded discharge. 


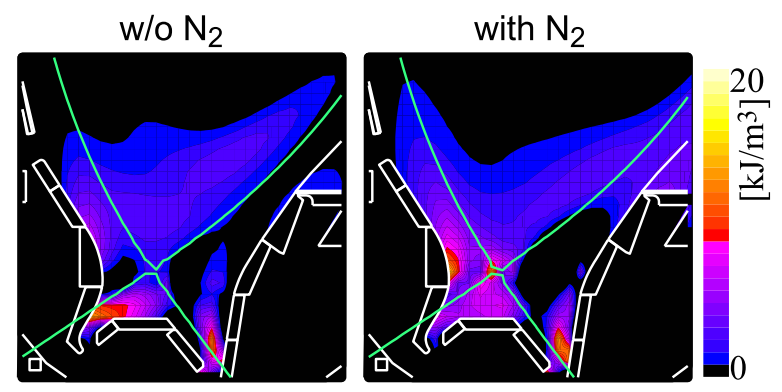

Figure 2: Distribution of the additional radiated energy due to a type-I ELM during a $2 \mathrm{~ms}$ interval around the ELM for the unseeded and the nitrogen seeded discharges from figure 1, averaged over several ELMs. 

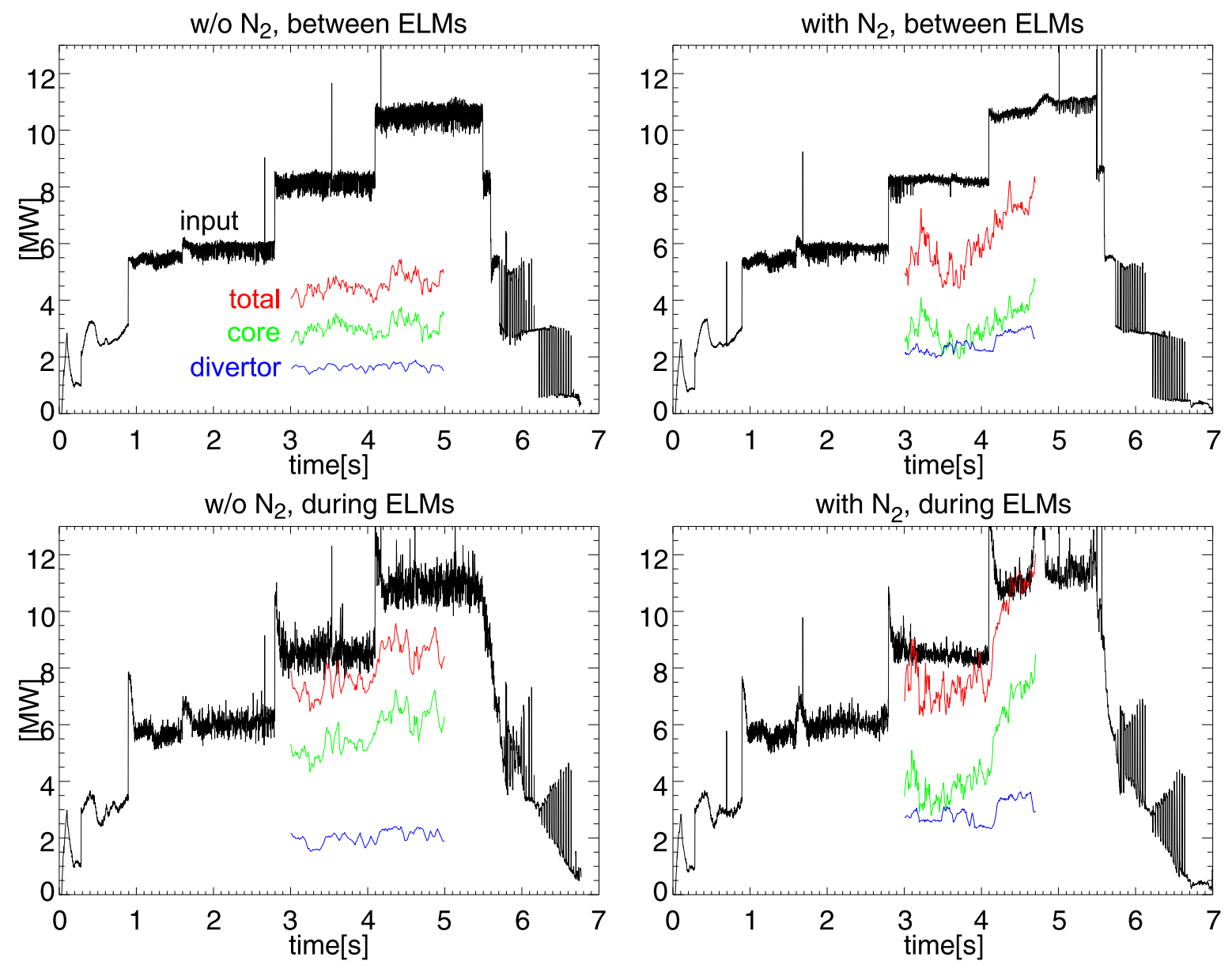

Figure 3: Time traces of the radiated power between (top) and during (bottom) ELMs for an unseeded (left) and nitrogen seeded (right) discharge. Plotted are the input power (black, heating power - $d W_{M H D} / d t$ ), the total radiated power, and the radiated power in the core plasma and in the divertor. 


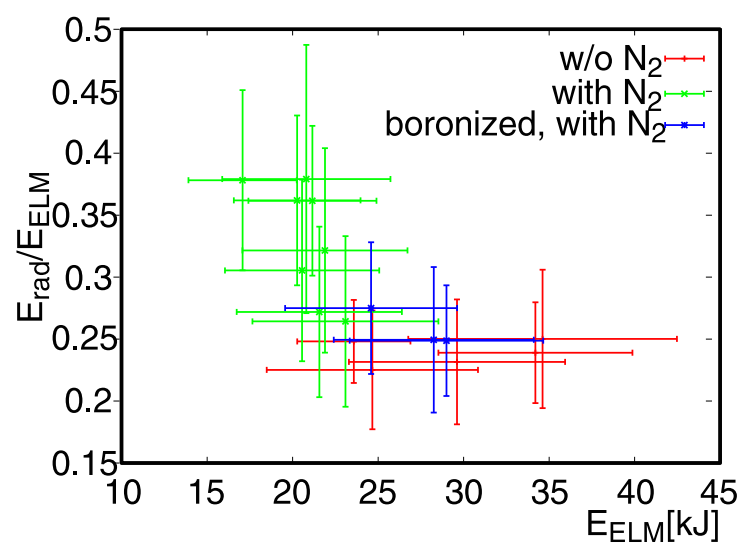

Figure 4: Dependence of the normalised ELM radiation $E_{r a d} / E_{E L M}$ (where $E_{\text {rad }}$ is the radiated energy due to a type-I ELM) of the ELM energy $E_{E L M}$ for unseeded discharges, nitrogen seeded discharges and nitrogen seeded discharges with boronised plasma facing components. Plotted are mean values for different shots with error bars. 

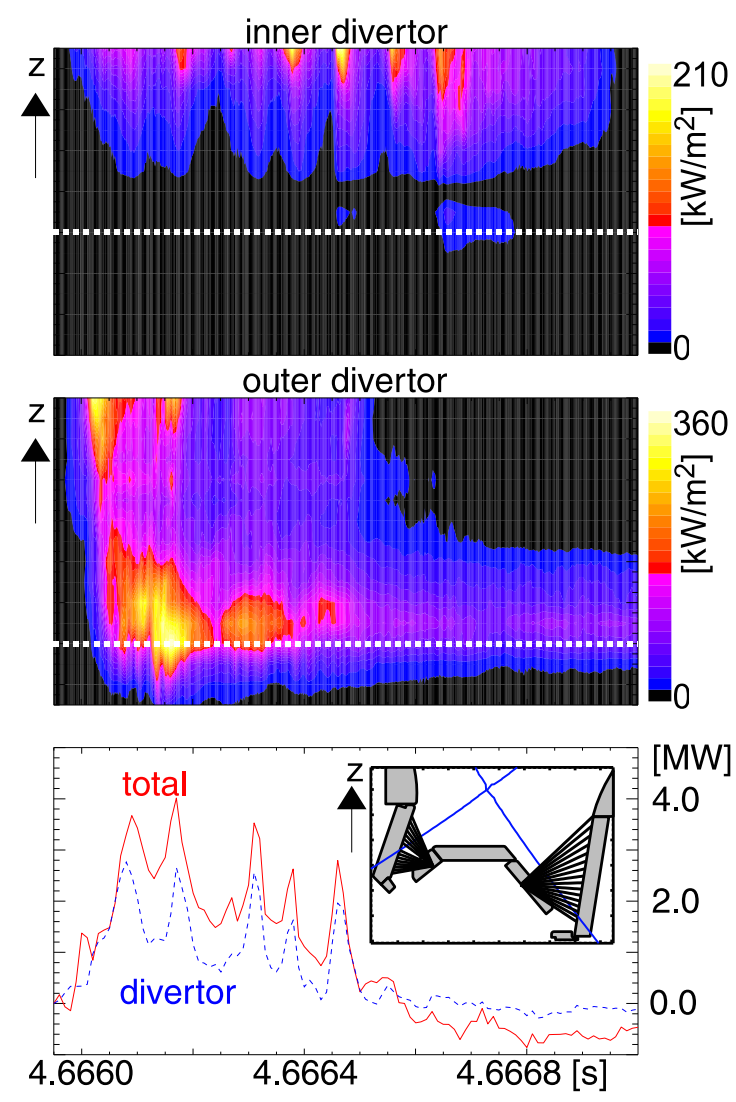

Figure 5: Development of the radiation during a type-I ELM. In the upper part contour plots of the line integrals of the AXUV diodes in the inner and outer divertor are shown, the position of these line of sights is shown in the inlay in the lower graph. The dotted line indicates the position of the strike points. The lower graph shows the total radiated power and the radiated power from the divertor due to the ELM (where the background radiation has been subtracted). 


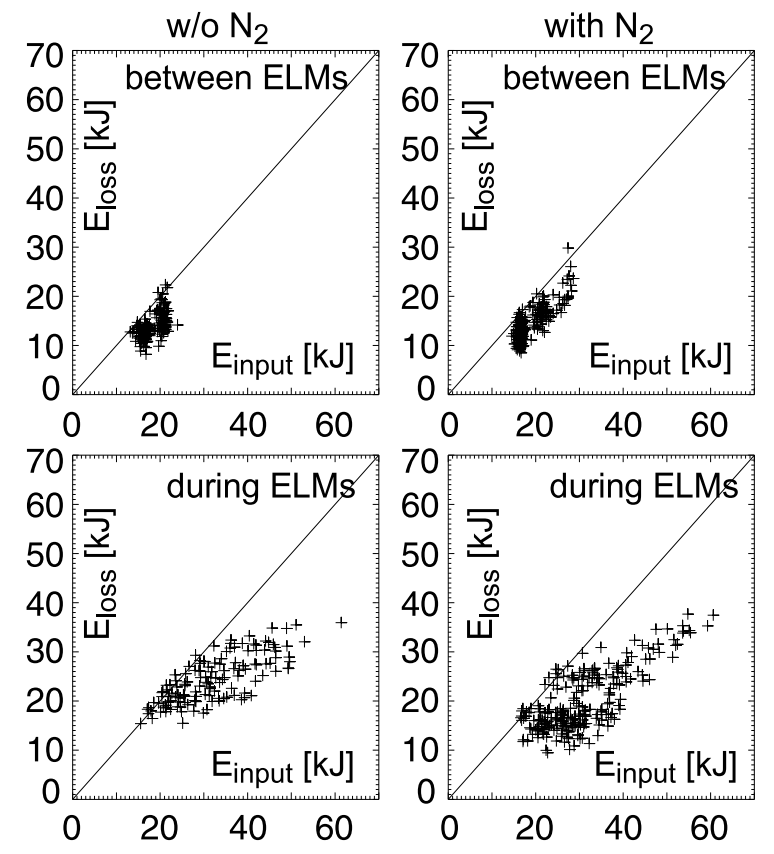

Figure 6: Energy balance between ELMs (top) and during ELMs (bottom) for unseeded (left) and nitrogen seeded (right) discharges. Plotted is the loss energy (radiated energy and energy load onto divertor target plates) during $2 m$ s intervals against the input energy (heating energy during the $2 m s$ interval and change of $W_{M H D}$ ). 